\title{
Paracetamol use, availability, and knowledge of toxicity among British and American adolescents
}

\author{
R J Gilbertson, E Harris, S K Pandey, P Kelly, W Myers
}

\begin{abstract}
Paracetamol is the commonest agent employed in self poisoning, however it is not clear whether adolescents possess insight into the serious complications associated with its misuse. Using a one page questionnaire, the availability, usage, and knowledge of toxicity of paracetamol among 1147 American and British adolescents was assessed. Although $90 \%$ of all students recognised that paracetamol could kill, the great majority of students overestimated the lethal dose. In addition, while knowledge regarding side effects of paracetamol was poor the drug was widely available to, and used by, the study population. It is proposed that gross overestimation of the number of tablets required to kill, poor understanding of paracetamol side effects, and wide availability of the drug contribute to its frequent use in adolescent suicidal behaviour. The inclusion of some over-thecounter medications in school drug education programs in addition to tighter control of the availability of paracetamol may help reduce the problem of adolescent self poisoning.

(Arch Dis Child 1996;75:194-198)
\end{abstract}

Keywords: adolescence, paracetamol, suicide.

Department of

Community

Paediatrics, Stanley

Health Centre, Stanley,

County Durham

R J Gilbertson

$S$ K Pandey

University

Department of Child

and Adolescent

Psychiatry, University of Florida, Gainsville, Florida, USA

H E Harris

W Myers

Department of Medical Statistics, Medical School,

University of

Newcastle upon Tyne

P Kelly

Correspondence to:

Dr R J Gilbertson, Cancer

Research Unit, Medical

School, Framlington Place,

University of Newcastle

upon Tyne, Newcastle upon

Tyne NE2 4HH.

Accepted 26 April 1996
Deliberate self poisoning among adolescents is a major health problem in Europe and the United States. ${ }^{1-3}$ Although recent total mortality statistics for the UK suggest an overall decline in the number of deaths from self poisoning, ${ }^{4}$ figures for adolescent deaths show a worrying increase among both British and American teenagers. In an early study of self poisoning teenage women in Edinburgh, Krietman and Schreiber reported a marked rise in hospital referrals from the late 1960 s to mid-1970s. ${ }^{5}$ Subsequent studies by Hawton's group in Oxford revealed a continuation in this upward trend with rates of overdose among 15-19 year old females increasing from 560/ 100000 in 1986 to $882 / 100000$ in $1989 .^{3} \mathrm{In}$ the United States the rate of recorded adolescent suicide has nearly tripled in recent decades, making it the second most common cause of death in those aged 15 to 24 years. $^{2}$ Recorded episodes of attempted suicide have also increased, with overdose being the most frequent method employed, accounting for $75 \%$ to $90 \%$ of cases. ${ }^{6}$

Both British and American adolescents most commonly employ readily available, over-thecounter medications in overdose. ${ }^{78}$ Changes in prescribing habits over the last 20 years have led to a decline in the availability of minor tranquillisers and sedatives while the simple analgesics have remained easily obtainable. ${ }^{9}$ In keeping with this, Hawton and Fagg have reported a considerable reduction in the use of minor tranquillisers by self poisoning adolescents, with a corresponding increase in paracetamol overdose. ${ }^{3}$ At a national level in the UK paracetamol is now the commonest agent used in self poisoning, ${ }^{10}$ and is the single most important cause of fulminant hepatic failure. ${ }^{11}$ During 1992, 78 deaths from poisoning occurred among individuals less than 20 years old, $27(35 \%)$ of which involved paracetamol. Of those deaths recorded as suicide, $50 \%$ involved paracetamol either exclusively, or in combination with other agents. ${ }^{12}$ These rates are similar to those reported in the United States where paracetamol is used by between $25 \%$ and $50 \%$ of self poisoning adolescents. ${ }^{13}{ }^{14}$

Although paracetamol is the commonest agent employed by adolescents in both suicidal behaviour and as a means of coping with intrapsychic or environmental stressors, it is not clear whether they possess insight into the potentially serious complications of such behaviour. A limited number of studies have attempted to address this question among overdose patients of all ages. In 1976 Gazzard et al published a study of 107 patients who had overdosed on paracetamol (the largest group of subjects falling in the 12 to 20 year old age range). Although all patients believed the overdose would be fatal and selected paracetamol because of its ready availability, their understanding of side effects was poor. All patients expected to 'lapse into coma' and less than $10 \%$ recognised hepatotoxicity as a potential danger. ${ }^{15}$ More recently Hawton et al surveyed 80 patients admitted to hospital in Oxford, England between September 1992 and March 1993 after paracetamol overdose. Once again the adolescent age group was the most frequently represented, knowledge of its lethality was high (more than $75 \%$ expecting overdose to cause death), and the availability of the drug the most frequent reason given for its use in overdose. ${ }^{8}$ These studies demonstrate that although adolescents are aware that paracetamol can kill, its availability and use in suicidal behaviour remains high.

In order to study this problem more closely in the adolescent age group Myers et al surveyed 169 American high school students in 1991 to assess their knowledge of paracetamol therapeutics, availability, side effects, and doses required to cause harm and death. In keeping with 'all age group' studies, $83 \%$ recognised the lethality of paracetamol overdose and $93 \%$ 
had access to the drug in their homes. However, a large proportion of those students recognising the lethality of paracetamol grossly overestimated the dose required to kill. ${ }^{16}$ Based on these results we propose that rather than underestimate the overall danger of this drug, adolescents place themselves at risk of paracetamol toxicity during suicidal gestures for three reasons: (1) paracetamol remains a readily available drug in significant quantities; (2) adolescents have a poor understanding of the side effects from overdose, for example selecting paracetamol for use in overdose believing excess ingestion will induce loss of consciousness; and (3) they grossly overestimate the number of tablets that will cause death. As a result, what may be considered relatively 'safe' for suicidal behaviour, for example $25,500 \mathrm{mg}$ tablets of paracetamol actually falls within the lethal dose range.

In the present study we have investigated the above hypothesis, expanding the earlier work of Myers et al to include 1147 British and American adolescents. The modified survey addresses the knowledge British and American adolescents have of paracetamol therapeutics and toxicity, their access to and general usage of paracetamol, and any cross cultural differences in knowledge between the British and American students.

\section{Subjects and methods}

A one page, 10 item questionnaire was developed from that used by Myers et al. ${ }^{16}$ The questionnaire included both multiple choice and open ended questions relating to: (1) demographic data, (2) therapeutic values of paracetamol, (3) availability at home and usage of paracetamol, (4) side effects, (5) potential for harm or lethality in overdose, and (6) quantity of drug to cause harm or death.

The questionnaire began with a section covering demographic data (age, school year/ grade, and sex). The availability of and indications for paracetamol were then assessed using three questions. The first addressed the indications for paracetamol: 'Paracetamol is used to treat (please circle the correct answer(s)): (i) vomiting/sickness, (ii) infections, (iii) high temperature/fever, (iv) pain/headaches, (v) to help sleep, and (vi) cold and flu'. High temperature/fever, pain/headaches, and cold and flu were judged correct answers. Next patients were asked to respond by circling the responses 'yes', 'no', or 'don't know' to the questions: 'Do you have paracetamol at home?' and 'Have you taken paracetamol within the last year?'

The final three questions related to the potential toxicity of paracetamol. First students were asked: 'Side effects (problems) from taking too much paracetamol are (please circle the correct answer(s)): (i) headache/ dizziness, (ii) sickness and vomiting, (iii) no side effects, (iv) sleepiness/sedation, (v) bleeding, (vi) fits/seizures, and (vii) damage to body parts/organs'. 'Sickness and vomiting' and 'damage to body parts/organs' were judged correct answers. In order to assess pupils beliefs as to whether paracetamol could harm or kill and the respective estimated doses to achieve such effects, students were asked two questions each with two parts. Question seven part (a) asked: 'Could someone take enough paracetamol to harm themselves? (please circle the correct answer)/yes or no' and part (b) 'If yes how many tablets in one go (each tablet is $500 \mathrm{mg}$ ) (please circle the correct answer)'. Students were offered a range of possible responses from five to 500 tablets, with answers below, within and above the 'toxic' and lethal doses. Finally, question eight assessed the students' perceptions of lethality. Part (a) asked: 'Could someone take enough paracetamol to kill themselves? (please circle the correct answer)/yes or no' and part (b) 'If yes how many tablets in one go (each tablet is $\mathbf{5 0 0}$ mg) (please circle the correct answer)', included the identical dose range used in question seven (b). Although children under 12 years demonstrate less hepatotoxicity than adults with equivalent toxic paracetamol plasma concentrations, adolescents develop hepatotoxicity within the range associated with liver damage in adults. ${ }^{17}$ The minimum toxic dose in adults is $125 \mathrm{mg} / \mathrm{kg}$, or approximately $10-15,500 \mathrm{mg}$ tablets in the age range studied. Answers to question seven (b) of 20 tablets or greater were therefore judged as 'overestimating' and 50 tablets or more 'grossly overestimating' the harmful dose. Lethal hepatotoxicity is common at a dose of $250 \mathrm{mg} / \mathrm{kg}$ or $20-30$, $500 \mathrm{mg}$ tablets for the study population. ${ }^{18}$ Answers to question eight (b) of 50 tablets or more were therefore judged as 'overestimating' the lethal dose and 100 or more 'grossly overestimating' the lethal dose.

Permission to conduct the study was obtained from the respective school headteachers in UK schools and, in the USA, from the University of Florida Institutional Review Board. The questionnaire was distributed by form class teachers and completed by students at the beginning of their first tutorial period. All questionnaires were distributed, completed, and collected on the same day. The UK study population was drawn from four comprehensive schools and one grammar school. USA students were surveyed from two high schools. Schools selected served both urban and rural communities and included students with a broad spectrum of social class backgrounds.

Comparisons between UK and USA student responses were made using $\chi^{2}$ statistics. The data were examined for linear trend across age ranges using the $\chi^{2}$ test for linear trend. The assumption of linearity was checked in all cases using the associated $\chi^{2}$ for non-linearity statistic. $^{19}$

\section{Results}

DEMOGRAPHIC DATA

Five hundred and eighty two UK students were surveyed. Age ranged from 13 to 16 years, with a mean of 14.8 years, $49.8 \% \quad(n=290)$ were male and $50.2 \%(n=292)$ female. School year ranged from year 9 to 11 with $46 \%(n=268)$ of students being year 11 . The USA study population included 565 students from two schools again serving a broad spectrum of social class 
Table 1 Age and sex distribution of students

\begin{tabular}{|c|c|c|c|c|c|c|}
\hline \multirow[b]{2}{*}{ Age (years) } & \multicolumn{3}{|c|}{ UK students } & \multicolumn{3}{|c|}{ USA students } \\
\hline & Male & Female & $\%$ Of total & Male & Female & $\%$ Of total \\
\hline 12 & 0 & 0 & 0 & 9 & 18 & 5 \\
\hline 13 & 31 & 37 & 12 & 89 & 86 & 31 \\
\hline 14 & 85 & 73 & 27 & 83 & 77 & 28 \\
\hline 15 & 87 & 94 & 31 & 37 & 32 & 12 \\
\hline 16 & 87 & 88 & 30 & 36 & 54 & 16 \\
\hline 17 & 0 & 0 & 0 & 19 & 21 & 7 \\
\hline 18 & 0 & 0 & 0 & 1 & 1 & 0.5 \\
\hline 19 & 0 & 0 & 0 & 0 & 2 & 0.5 \\
\hline
\end{tabular}

groups, with a slightly broader age range of 12 to 19 years but similar mean age of 14.3 years. Again the sex distribution was equal with $48.5 \%(n=274)$ male and $51.5 \%(n=291)$ female students (table).

\section{AVAILABILITY, USE, AND THERAPEUTIC}

INDICATIONS

No significant effect of age, sex, school year/ grade, or nationality was seen in the manner students responded to question three, assessing knowledge of the therapeutic value of paracetamol. Ninety eight per cent $(n=553)$ and $86 \%$ $(n=484)$ of students from the UK and USA respectively, recognised the use of paracetamol to treat pain, while $22 \%$ of American and $31 \%$ of British students marked 'cold and flu' as a further area of use for paracetamol. Access to the drug was almost universal with $94 \% \quad(n=549)$ of students from the UK and $86 \%(n=486)$ of those from the USA reporting to have paracetamol at home with no sex or age difference. Usage of paracetamol was also common for all ages. However whereas $89 \%$ of American students had taken paracetamol during the previous 12 months with no age difference $\left(\chi^{2}=8.6, p=0.23\right)$, British students revealed a stepwise increase in usage with increasing age, from $76 \%$ of 13 year olds to $93 \%$ of 16 year olds $\left(\chi^{2}\right.$ for trend $=14.0, p$ $<0.0001$; fig 1 ).

SIDE EFFECTS AND TOXICITY

With regard to the potential side effects of paracetamol, a significant difference in the

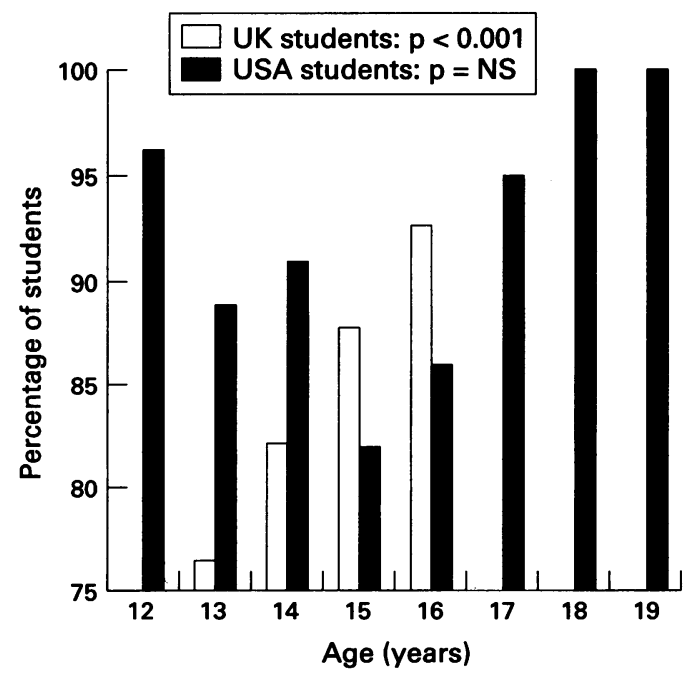

Figure 1 Percentage of students from the UK and USA taking paracetamol within the previous year by age. $A$ significant increase in use with increasing age was seen among UK students but not in those from the USA. level of knowledge at differing ages was observed between students from the UK and USA. Those from the UK demonstrated increasing knowledge with age, with $6 \%$ of 13 year olds and $22 \%$ of 16 year olds recognising both 'sickness and vomiting' and 'organ damage' as true adverse effects $\left(\chi^{2}=15.2\right.$, $\mathrm{p}<0.0001$ ). In contrast an inverse relationship to age was observed in those from the USA falling from $18 \%$ of 12 year olds marking both correct answers to $2 \%$ of 17 year olds $\left(\chi^{2}=4.8\right.$, $\mathrm{p}=0.03$; fig 2). However, one of the two 18 year olds and both 19 year old USA students correctly identified the two appropriate answers.

A similar pattern of response was observed when we analysed all students identifying 'damage to body parts/organs' as an adverse effect regardless of their other responses to this question (fig 3). Students from the UK again revealed a significant increase in knowledge with age $\left(\chi^{2}=15.6, \mathrm{p}<0.0001\right)$. However while those from the USA demonstrated a trend toward an inverse relationship between age and knowledge this did not reach significance, $\chi^{2}=3.5, \mathrm{p}=0.06$.

Belief that parcetamol could cause sedation was common among both British and Ameri-

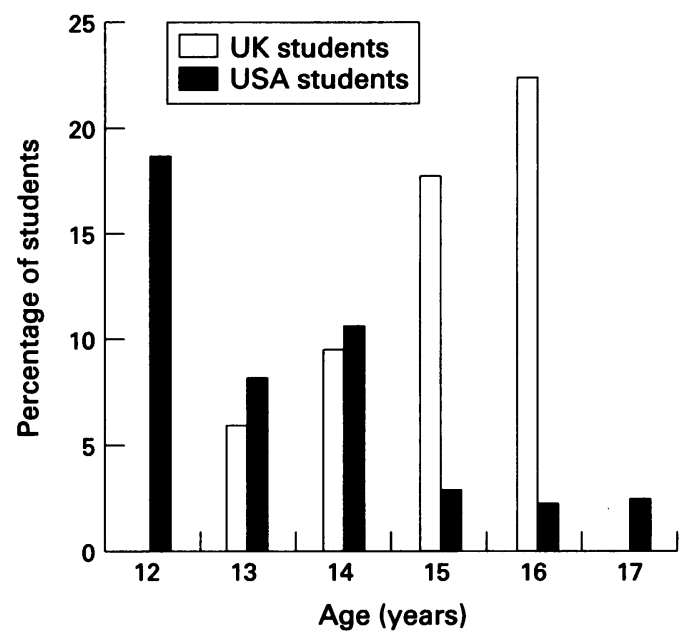

Figure 2 Age distribution of students from the UK and USA marking organ damage and vomiting as side effects of excess paracetamol ingestion.

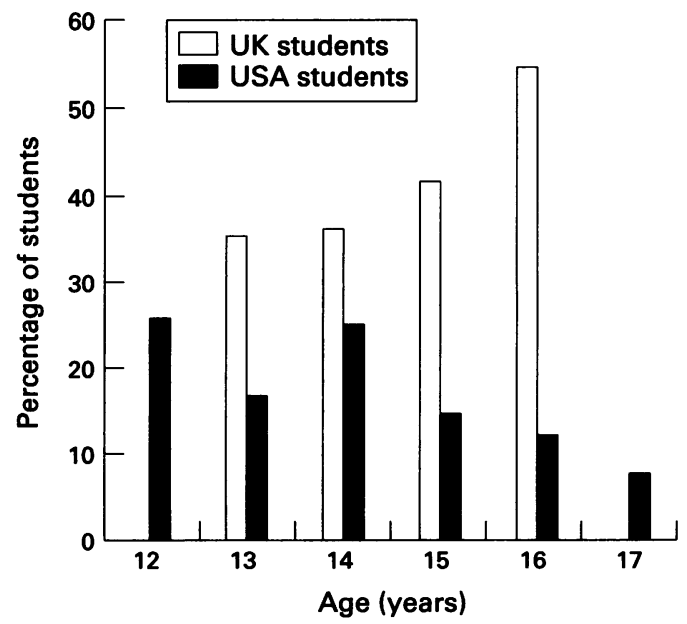

Figure 3 Age distribution of students from the UK and USA marking organ damage as a side effect of excess paracetamol ingestion. 
can students (fig 4). However more of those from the UK incorrectly identified 'sleepiness and sedation' as a side effect than USA students. In addition the frequency of this erroneous response increased with age from $24 \%$ at 12 years to $46 \%$ at 16 years $\left(\chi^{2}=9.5, p\right.$ $=0.002)$. The reverse pattern was identified in USA students with approximately half the number of 17 year olds (17\%) marking sedation as a potential side effect than 12 year olds (30\%); this however did not reach significance $\left(\chi^{2}=2.88, p=0.09\right)$.

Finally, with regard to whether pupils believed paracetamol could harm or kill, no significant effect of age, sex, school year/grade, or nationality was seen in the manner students responded to questions seven and eight. Ninety nine per cent $(n=577)$ of students from the UK and $94 \%(n=534)$ of those from the USA answered that paracetamol in sufficient quantities could 'harm' you. In response to number of tablets to cause 'harm' almost identical 'toxic dose' distributions were seen for UK and USA questionaires (fig 5). Fifty eight per cent of British and $41 \%$ of American students overestimated the 'harmful' dose stating that 20 or more $500 \mathrm{mg}$ tablets were required to cause harm. Of students answering 20 tablets or more, approximately half of those from both the UK and USA grossly overestimated the harmful dose, stating $\mathbf{5 0}$ tablets or more were required to cause harm. With respect to lethality, again almost all UK $(97 \%, n=564)$ and USA ( $89 \% \mathrm{n}=500)$ students recognised that paracetamol in overdose could kill. Dose overestimation was even higher than that for dose to 'harm' (fig 6). Seventy five per cent of students from the UK and $62 \%$ of those from the USA overestimated the lethal dose (answering 50 or more tablets to kill) while $45 \%$ of UK and $41 \%$ of USA students respectively grossly overestimated the dose of paracetamol required to kill (answering 100 or more tablets to kill).

\section{Discussion}

Paracetamol is employed by a high proportion of self poisoning adolescents, ${ }^{12-14}$ and in the UK is the most frequently used medicine in

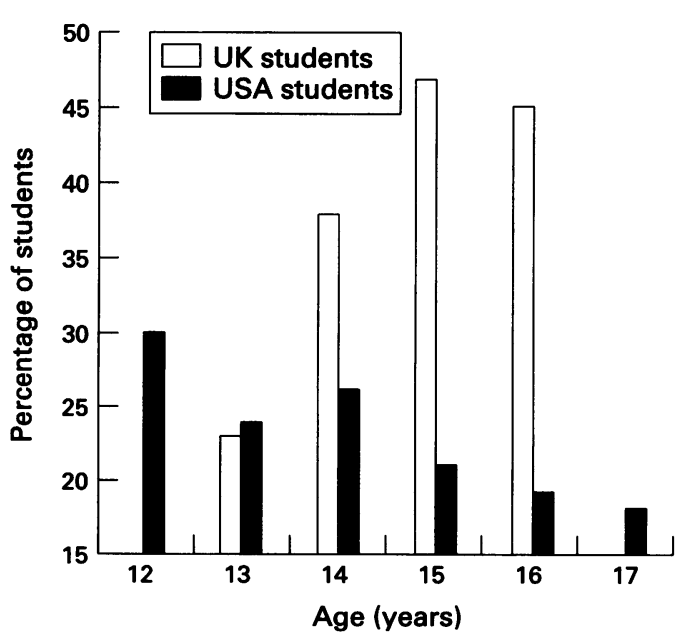

Figure 4 Age distribution of students from the UK and USA believing sedation to be a side effect of paracetamol. self poisoning. ${ }^{10}$ This worrying trend has continued despite increased public awareness of its lethality in overdose. ${ }^{816}$ We propose that adolescents continue to place themselves in jeopardy of paracetamol toxicity for three reasons: (1) paracetamol remains a readily available drug in significant quantities, (2) adolescents have a poor understanding of the side effects from overdose, and (3) they grossly overestimate the number of tablets that will cause death.

The present study confirms that paracetamol is widely used by and available to both British and American adolescents. Students in the UK demonstrated a stepwise increase in usage with increasing age (fig 1), while level of paracetamol use by USA students was high for all ages surveyed. Although not surprising, these results confirm that paracetamol is a familiar drug to the adolescent, supporting the hypothesis that availability may contribute to its abuse in self poisoning.

In addition to availability, confusion regarding the true side effects of the drug may also contribute to its frequent use in overdose. For example, despite a paucity of symptoms in the acute phase of intoxication, many self poisoning patients take paracetamol believing it will

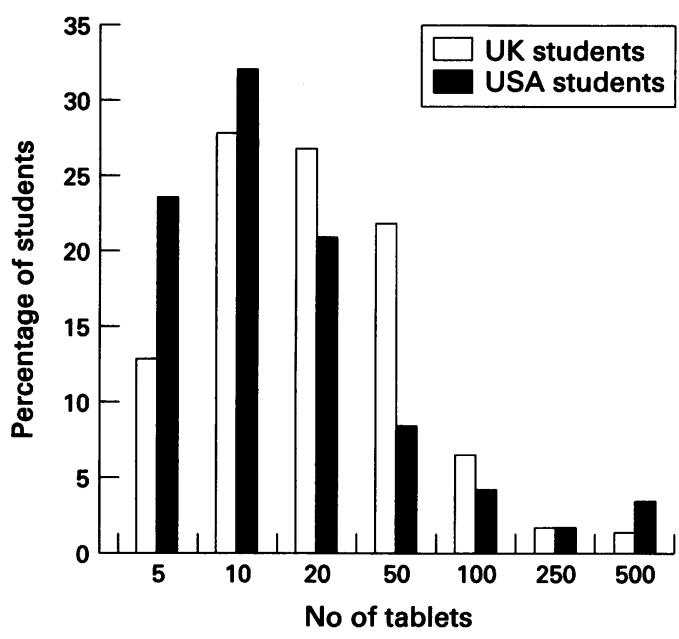

Figure 5 Dose distribution of responses from students from the UK and USA in answer to the question 'dose required to cause harm'.

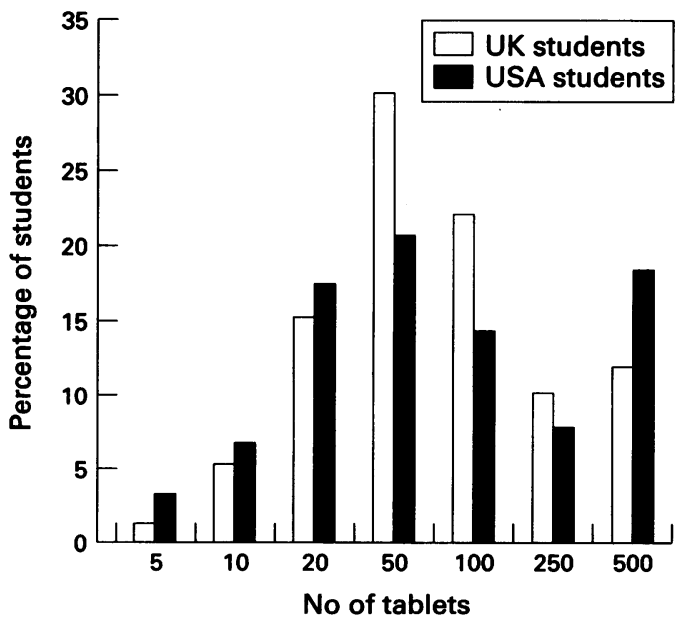

Figure 6 Dose distribution of responses from students from the UK and USA in answer to the question 'dose required to kill'. 
induce loss of consciousness. ${ }^{815}$ In the present study $23 \%$ of 12 year old and $46 \%$ of 16 year old students in the UK believed 'sleepiness/ sedation' could result from excessive ingestion of the drug. It is not clear why a greater proportion of older than younger UK students should hold this erroneous belief. However it is of concern that this occurs more frequently in the older teenage years when paracetamol self poisoning is more common. ${ }^{12}$ Furthermore this poor understanding of the true toxicity profile of paracetamol may also have a deleterious effect on the clinical outcome of overdose. This may result from delayed presentation of the adolescent to medical services because of an absence of erroneously expected acute toxicity. Such delay would significantly reduce the likelihood of receiving early, effective medical treatment.

Although the majority of both British and American students had little understanding of the true side effects of paracetamol, students in the UK did demonstrate a significant increase in knowledge with age. Six per cent of 13 year old UK students and $22 \%$ of 16 year olds respectively recognised both 'sickness and vomiting' and 'organ damage' as adverse effects, $\chi^{2}=15.2, \mathrm{p}<0.0001$ (fig 2). A similar pattern of response was observed when we analysed all students identifying 'damage to body parts/organs' as an adverse effect regardless of their other responses to this question $\left(\chi^{2}=15.6, \mathrm{p}<0.0001\right)$. In contrast, students in the USA demonstrated a significant, inverse relationship between age and knowledge of paracetamol side effects (fig 3 ). This surprising apparent decline in knowledge with increasing age may reflect a recent drug education program aimed at the younger members of the American study population.

Perhaps the most worrying results from the present study relate to questions seven and eight assessing pupils' belief as to whether paracetamol could harm or kill. In keeping with the proposed hypothesis, although the majority of British and American students recognised that in sufficient quantities paracetamol could 'harm' you, $58 \%$ of students in the UK and $41 \%$ of those in the USA overestimated the 'harmful' dose. Of these students, half made gross overestimations. Similar results were observed with respect to lethality; again almost all the British and American students recognised that paracetamol in overdose could kill with $75 \%$ of UK and $62 \%$ of USA students overestimating the lethal dose and $45 \%$ of UK and $41 \%$ of USA students respectively grossly overestimating the dose of paracetamol required to kill (answering 100 or more tablets to kill).

We believe that widespread availability and poor understanding of its true side effects and toxicity significantly influences the use of paracetamol by adolescents in self poisoning. In addition lack of symptoms in the acute phase of toxicity may delay the presentation of self poisoning adolescents to the medical services. Since the completion of this study Hawton $e t$ al have published the results of their survey of 80 consecutive paracetamol overdose patients. In this study availability of the drug significantly influenced self poisoning behaviour with $37 \%$ stating they would have taken a smaller overdose or none at all, if fewer tablets had been available. Moreover, only $35 \%$ said they would have taken the overdose had they known of the lack of acute side effects such as loss of conciousness. ${ }^{20}$

We propose that in addition to the problem of illicit substances, drug education programs in schools should address the dangers of overthe-counter medications. In addition we support recent calls for tighter control of the availability of paracetamol ${ }^{8}{ }^{20}$ or the inclusion of antidote in paracetamol preparations.

Thanks are due to the pupils and staff of the four British and two American schools involved in the study.

1 Diekstra RFW. Suicide and suicide attempts in the European Economic Community: an analysis of trends with special emphasis upon trends among the young. Suicide Life Threat Behav 1985; 15: 27-42.

2 Rosenberg ML, Smith JC, Davidson LE, Conn JM. The emergence of youth suicide: an epidemiological analysis and public health perspective. Annu Rev Public Health 1987; 8: 417-27.

3 Hawton K, Fagg J. Deliberate self-poisoning and self-injury in adolescents. $A$ study of characteristics and trends in Oxford, 1976-1989. Br ₹ Psychol 1992; 161: 816-23.

4 Spooner JB. Paracetamol and self poisoning. BMF 1995; 310: 1072 .

5 Krietman $\mathrm{N}$, Schreiber M. Parasuicide in young Edinburgh women. Psychol Med 1979; 9: 469-79.

6 Spirito A, Brown L, Overholser J, Fritz G. Attempted suicide in adolescence: a review of the literature. Clin Psychol Rev 1989; 9: 335-63.

7 Morgan HG, Burns-Cox CJ, Pocock HJ, Pottle S. Deliberate self-harm: clinical and socioeconomic characteristics of 368 patients. Br $\mathcal{P}$ Psychiatry 1970; 127: 564-74.

8 Hawton K, Ware C, Mistry H, et al. Why patients choose paracetamol for self-poisoning and their knowledge of its dangers. $B M \Im$ 1995; 310: 164 .

9 Forster DP, Frost CEB. Medicinal self-poisoning and prescription frequency. Acta Psychiatr Scand 1985; 71: 657-74.

10 Manufacturing Chemists Association/Committee on Safety of Medicines. Reminder: paracetamol toxicity in overdose. of Medicint Problems in Pharmacovigilance 1995; 21: 10.

11 Mutimer DJ, Ayers RCS, Neuberger JM, et al. Serious paracetamol poisoning and the results of liver transplantation. Gut 1994; 35: 809-14.

12 Office of Population Censuses and Surveys. Mortality statistics: injury and poisoning 1992. London: HMSO, 1994: table 10.

13 Veltri JC, Rollins DE. A comparison of the frequency and severity of poisoning cases for ingestion of acetaminophen, asprin and ibuprofen. Am $\mathcal{F}$ Emerg Med 1988; 6: 104-7.

14 Ashbourne JF, Olson KR, Khayam-Bashi $\mathrm{H}$. Value of rapid screening for acetaminophen in all patients with intentional drug overdose. Ann Emerg Med 1989; 18: 1035-8.

15 Gazzard BG, Davis M, Spooner J, Williams R. Why do people use paracetamol in overdose? $B M F 1976$; i: $212-3$.

16 Myers WC, Otto TA, Harris E, Diaco D, Moreno A. Acetaminophen overdose as a suicidal gesture: a survey of adolescents' knowledge of its potential for toxicity. $\mathcal{f} \mathrm{Am}$ Acad Child Adolesc Psychiatry 1992; 31: 686-90.

17 Ellenham M, Barceloux J, eds. Acetaminophen (paracetamol). Medical toxicology. Elsevier, 1988: 156-66.

18 Prescott LF, Critchley JAJH. The treatment of acetaminophen poisoning. Annu Rev Pharmacol Toxicol 1983; 23: nophen

19 Armitage R, Berry $\mathbf{M}$, ed. Statistical methods in medical Armitage $\mathbf{R}$, Berry $\mathbf{M}$, ed. Statistical methods
research. Oxford: Blackwell Scientific, 1994.

20 research. Oxford: Blackwell Scientific, 1994. self-poisoning. Characterstics, prevention and harm reducself-poisoning. Characterstics, preven
tion. Br $\mathcal{F}$ Psychiatry 1996; 168: 43-8. 